\title{
Healing-Promoting Action of Teprenone, a New Antiulcer Agent on Acetic Acid Ulcer in Rats
}

\author{
Mikio ITO, Akira FUJII and Yoshio SUZUKI \\ Department of Pharmacology. Faculty of Pharmacy, Meijo University. \\ Tenpaku-ku, Nagoya 468 , Japan \\ Accepted April 8, 1985
}

\begin{abstract}
Healing promoting action of teprenone on acetic acid ulcer in rats was assessed in comparison to that of cimetidine and proglumide by histological measurements. Teprenone $(100 \mathrm{mg} / \mathrm{kg} \times 2 /$ day, p.o.) decreased the macroscopic ulcer index and the defective area in the ulcerated region by $32.0 \%$ and $33.3 \%$, respectively. In addition, this drug increased the decreasing index of exposed ulcer floor and the mucosal regeneration index by $28.1 \%$ and $38.0 \%$, respectively. However, the thickness of the ulcer base and the development index of collagen fibers were little affected by this drug. Cimetidine (100 $\mathrm{mg} / \mathrm{kg} \times 2 /$ day, p.o.) showed a $27.2 \%$ decreasing action on the macroscopic ulcer index and a $31.3 \%$ increasing action on the thickness of the ulcer base, but failed to increase the mucosal regeneration index. Specimens from the cimetidine-treated rats were characterized by marked granulation proliferation beneath the ulcer floor. The upper layer of the granulation tissue was very rich in inflammatory cells. Proglumide $(250 \mathrm{mg} / \mathrm{kg} \times 2 / \mathrm{day}, \mathrm{p.o}$.) was scarcely effective on any parameters. These results indicate that teprenone remarkably promotes the regeneration of the defective mucosa during the ulcer healing process, and the effectiveness of cimetidine according to the macroscopic observation may be due to excessive formation of granulation tissue in the defective region.
\end{abstract}

It has been aiready demonstrated by Murakami et al. (1) that teprenone (geranylgeranylacetone), an acyclic polyisoprenoid, has marked antiulcer effects on several types of experimentally induced peptic ulcers. It has been also indicated as biochemical characteristics of the antiulcer actions of teprenone that this drug has no effect on gastric secretion in pylorus-ligated rats (1). but that this drug can inhibit the decrease in hexosamine, an indicator of gastric glycoprotein, in the gastric mucosa of rats subjected to cold-restraint stress (2,3) and the decrease in gastric macromolecular glycoprotein following oral administration of aspirin to rats (4).

On the other hand, authors previously followed the healing process of acetic acid ulcer in rats over a long period of time by histological measurements and reported that these measurements can quantitatively evaluate histological changes such as the regeneration of defective mucosa and the proliferation of granulation tissue formed in the defective region (5)

The present study was designed to evaluate the healing-promoting action of teprenone on acetic acid ulcer in rats by histological measurements. Furthermore, the effects of teprenone were compared with those of cimetidine, a histamine $\mathrm{H}_{2}$ receptor antagonist, and proglumide, a gastrin antagonist (6)

\section{Materials and Methods}

Animals: Male Sprague-Dawley strain SPF rats weighing approx. $180 \mathrm{~g}$ (Shizuoka Agricultural Cooperative Association for Laboratory Animals, Shizuoka) were used. These animals were housed in an airconditioned room at $23 \pm 1^{\circ} \mathrm{C}$

Drugs: Drugs used were teprenone 
(geranylgeranylacetone, 6, 10, 14, 18-tetramethyl-5, 9.13.17-nonadecatetraene-2-one; Eisai, Co., Ltd.), cimetidine (was provided by Eisai, Co.. Ltd.) and proglumide (was provided by Eisai, Co., Ltd.). Teprenone was emulsified in 5\% gum arabic and $0.6 \%$ Tween 80, and the other two drugs were suspended in these detergents.

Ulcer induction: Gastric ulcer was induced in rats by the injection of $20 \%$ acetic acid in a volume of $0.05 \mathrm{ml}$ into the serosal layer in the glandular part of the stomach in accordance with the method described by Takagi et al. (7).

Drug administration: Each test drug, emulsified or suspended, was given orally. twice a day in a volume of $1 \mathrm{ml} / 100 \mathrm{~g}$ of body weight for 14 consecutive days beginning the day of acetic acid injection. Control animals were given only the vehicle instead of test drugs. The effects of test drugs were evaluated on the 15th day by the method described below.

Macroscopic measurement: On the 15th day, the animals were sacrificed by rapid decapitation. The stomachs were removed, filled with $5 \mathrm{ml}$ of $10 \%$ formalin and allowed to stand for $5 \mathrm{~min}$. Then, the stomachs were cut open along the greater curvature and spread on a gum plate. The longitudinal and abscissal lengths of ulcer were measured with a caliper using a magnifying glass, and the product of both lengths $\left(\mathrm{mm}^{2}\right)$ was expressed in terms of the macroscopic ulcer index.

Histological measurements: Histological measurements were carried out in accordance with the modification of the method reported by Tabayashi (8). After measuring the macroscopic ulcer size, the stomach tissue was again immersed in 10\% formalin for $24 \mathrm{hr}$ and then washed in running tap water for 12 hr. After washing, the tissue was dehydrated by immersing it stepwise into low to high concentrations of alcohol. The formalin-fixed tissue was cut so that a little of the normal tissue surrounding the ulcer remained. Thereafter, the central part of ulcer was cut vertically against the serosa along the long diameter. These tissues cut in half were embedded in paraffin and cut into 2-3 $\mathrm{l} / \mathrm{m}$ thick sections. The sections were stained with hematoxylin and eosin (HE) and Masson trichrome (MT). Histological measurements were performed under light micrography of

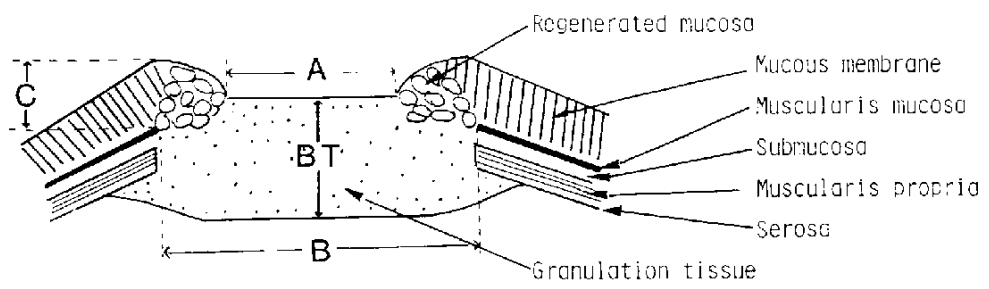

Measurements of ulcerated region

A: Defect of : iucoso

B: Extent of runtured muscularis mucoso

C: Height of marginal mucosa

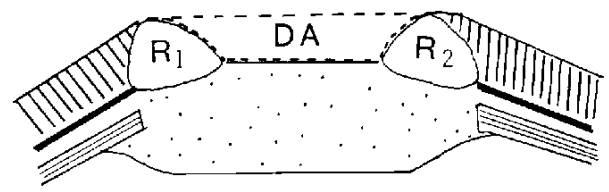

BT; Thickness of uicer bose

DA: Lefective area in ulcerated region

$R_{1}+R_{2}$ : Area of regeneroted mucoso

colculation of indices

Lecreosing index of exposed ulcer floor: $\frac{B-A}{B} \times 100$

Mucosal regeneration index: $\frac{R_{1}+R_{2}}{C \times B} \times 100$

Fig. 1. Method for histological measurements. 
HE-stained preparations as shown in Fig. 1. Of the data measured here, the thickness of the ulcer base and the defective area in the ulcerated region were used for evaluating the effect of test drugs. Furthermore, the decreasing index of exposed ulcer floor and the mucosal regeneration index were calculated on the basis of the measured data. In this case, the decreasing index of exposed ulcer floor is designated as 100 when the ulcer floor is completely covered with the regenerated mucosa. On the other hand, the mucosal regeneration index is desigrated as 100 when the ulcer floor is completely covered with the regenerated mucosa equal to the marginal mucosa of the ulcer in height. Then, the development index of collagen fibers (CDI) was calculated in accordance with the classification of Tabayashi (8) under light microscopic observation of the MT. stained preparations as follows: CDI 0 : Although there are a large number of fibroblasts and blood capillaries, no collagen fiber is recognized in the ulcer base. CDI 20: The fibrization of granulation tissue begins and several collagen fibers can be seen in a visual fields. CDI 40: Collagen fibers can be seen in $1 / 3$ of a visual field. CDI 60 : Collagen fibers can be seen in $1 / 2$ of a visual field. CDI 80: The fibrization develops and the ulcer base reveals a network of a large number of collagen fibers. CDI 100: The cicatrization is completed by innumerable collagen fibers, and blood capillaries and fibroblasts can be seen no longer.

Statistical analysis: Results obtained were expressed as the mean \pm S.E. Student's $t$-test was used for statistical analysis.

\section{Results}

Macroscopic and histological measurements: As shown in Fig. 2. teprenone at doses of 50 and $100 \mathrm{mg} / \mathrm{kg} \times 2 /$ day, p.o. significantly decreased the macroscopic ulcer index by $34.0 \%$ and $32.0 \%$, respectively. In addition, at a dose of $100 \mathrm{mg} / \mathrm{kg} \times 2 /$ day, p.o., this drug significantly decreased the defective area in the ulcerated region by $33.3 \%$ and increased the decreasing index of exposed ulcer floor and the mucosal regeneration index by $28.1 \%$ and $38.0 \%$, respectively. However, the thickness of the ulcer base and the development index of collagen fibers were little affected even by a high dose of this drug. Thus, teprenone (100 $\mathrm{mg} / \mathrm{kg} \times 2 /$ day, p.o.) remarkably promoted the regeneration of the defective mucosa without affecting the thickness of the ulcer base which indicates the degree of granulation proliferation beneath the ulcer
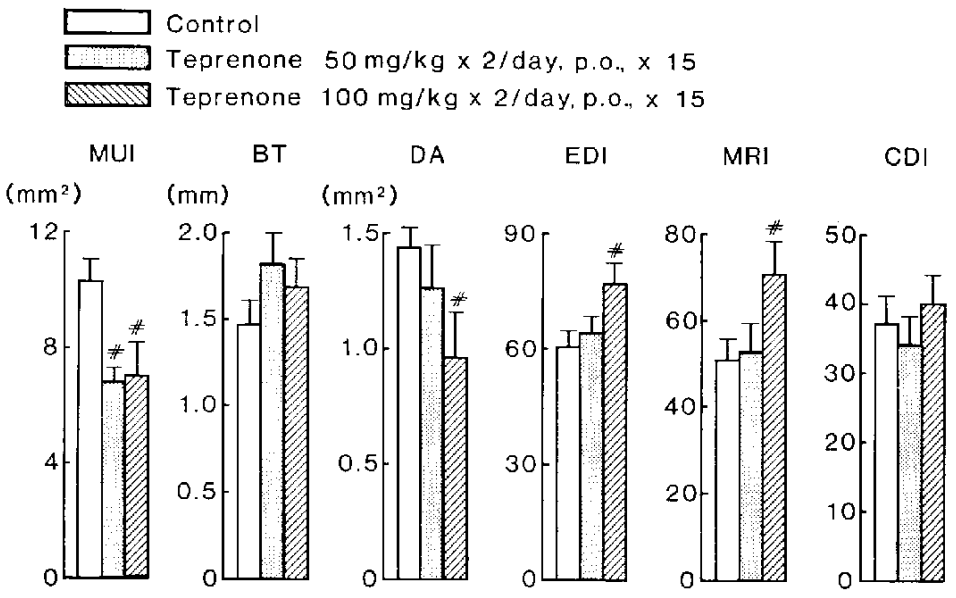

Fig. 2. Effects of teprenone on the healing of acetic acid ulcer in rats. Each column denotes the mean value with S.E. (Control: $N=14$, Test drug: $N=10$ or 11). MUl: Macroscopic ulcer index. BT: Thickness of ulcer base. DA: Defective area in ulcerated region. EDI: Decreasing index of exposed ulcer floor. MRI: Mucosal regeneration index. CDI: Development index of collagen fibers. $\because P<0.05$, compared with the control. 
floor.

The result for cimetidine is given in Fig. 3 . Cimetidine at a doses of $100 \mathrm{mg} / \mathrm{kg} \times 2 /$ day. p.o., resulted in a $27.2 \%$ significant decrease of the macroscopic ulcer index and a $31.3 \%$ significant increase of the thickness of the uicer base, although it was ineffective at a dose of $50 \mathrm{mg} / \mathrm{kg} \times 2 /$ day, p.o. Cimetidine at a low dose tended to increase the development index of collagen fibers. However, this drug at both doses showed no significant effects on the decreasing index of exposed ulcer floor and the mucosal regeneration index. Thus, it is very interesting that cimetidine $(100 \mathrm{mg} / \mathrm{kg} \times 2 /$ day, p.o.) failed to promote the mucosal regeneration, although this drug stimulated the formation of granulation tissue beneath the ulcer floor.

On the other hand, proglumide (250 mg/ $\mathrm{kg} \times 2 /$ day, p.o.) was hardly effective on the macroscopic and histological parameters measured as illustrated in Fig. 4.

Histological findings: In the control group. there was no cases in which the ulcer floor was completely covered with the regenerated mucosa, although a marked formation of

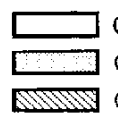

Control

Cimetidine $50 \mathrm{mg} / \mathrm{kg} \times 2 /$ day. p.0., $\times 15$

Cimetidine $100 \mathrm{mg} / \mathrm{kg} \times 2 /$ day, p.0., $\times 15$

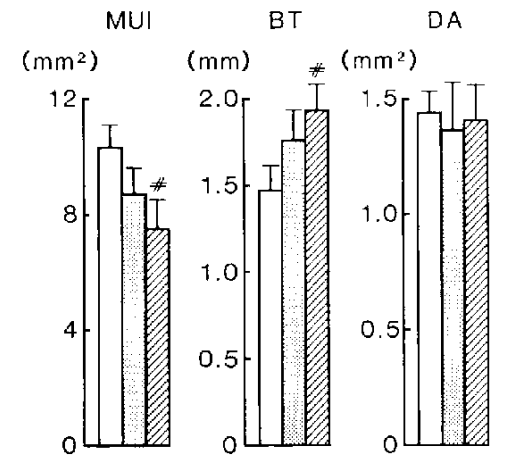

EDI

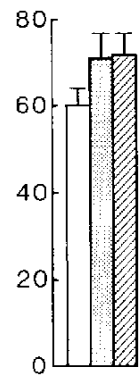

MRI

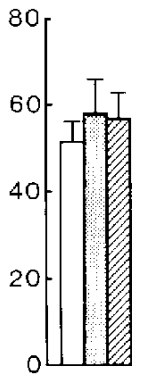

CDI

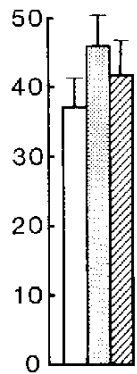

Fig. 3. Effects of cimetidine on the healing of acetic acid ulcer in rats. The control is the same as that of teprenone. Each column denotes the mean value with S.E. (Control: $N=14$, Test drug: $N=10$ or 11 ). $₫ P<0.05$, compared with the control. For other references, see legend to Fig. 2.

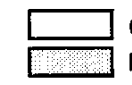

Control

Proglumide $250 \mathrm{mg} / \mathrm{kg} \times 2 /$ day, p.0., $\times 15$
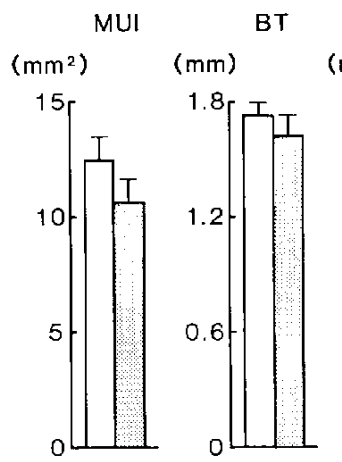

$\mathrm{DA}$

EDI
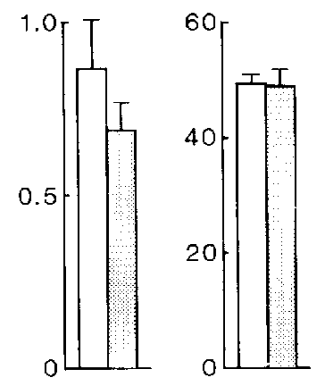

MRI

CDI
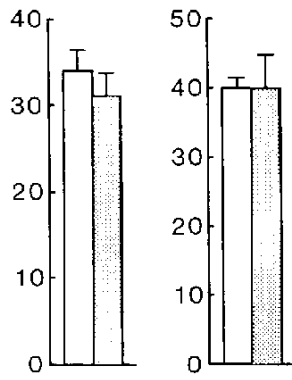

Fig. 4. Effects of proglumide on the healing of acetic acid ulcer in rats. Each column denotes the mean value with S.E. (Control: $N=11$, Test drug: $N=11$ ). For other references, see legend to Fig. 2. 
granulation tissue was seen in the defective region. In this case, the regenerated mucosa which consists mainly of pseudopyloric glands was slightly observed on the marginal region of the ulcer floor.

In the teprenone $(100 \mathrm{mg} / \mathrm{kg} \times 2 /$ day, p.o.) treated group, the regeneration of defective mucosa was remarkable and in 4 out of 11 cases, the ulcer floor was almost completely covered with the regenerated mucosa.

On the other hand, the specimens obtained from rats of the cimetidine $(100 \mathrm{mg} / \mathrm{kg} \times 2$ / day, p.o.)-treated group were characterized by a marked granulation proliferation beneath the ulcer floor. In the cases showing severe granulation proliferation, the regeneration of defective mucosa was poor. In this group. the upper laver of the granulation tissue was very rich in inflammatory cells as compared with that of the control group.

Histological findings of the specimens from rats of the proglumide $(250 \mathrm{mg} / \mathrm{kg} \times 2 /$ day, p.o.)-treated group were scarcely different from those of the control group.

\section{Discussion}

In the present study, teprenone and cimetidine showed different effects on the healing-promoting action of acetic acid ulcer in rats. Teprenone (100 $\mathrm{mg} / \mathrm{kg} \times 2 / \mathrm{day}$, p.o.) remarkably promoted the regeneration of the defective mucosa without affecting the granulation proliferation beneath the ulcer floor. On the other hand. cimetidine (100 $\mathrm{mg} / \mathrm{kg} \times 2 /$ day, p.o.) stimulated the formation of granulation tissue with a marked cell infiltration.

It has been considered that teprenone may exert the antiulcer actions through increasing the defensive force of the gastric mucosa by preventing the decrease in gastric macromolecular glycoprotein in aspirin- or cold restraint-induced ulcers of rats $(3,4)$. Murakami et al. (9) investigated the effect of teprenone on kinetics of mucus secreting cells in mouse gastric mucosa by using an autoradiographic technique with ${ }^{3} \mathrm{H}$ thymidine and found out that this drug prevented the reduction of the labeled cells in the fundic glands following the treatment with hydrocortisone, an ulcerogenic agent. Recently, authors reported that a glycoprotein having a molecular weight of less than $2.0 \times 10^{6}$, which lost much of its viscous and gel-forming properties, increased in the gastric mucus obtained from both ulcerated and non-ulcerated portions during the healing process in acetic acid uicer in rats (10). From these findings, therefore, it is suggested that teprenone may promote the regeneration of defective mucosa during the ulcer healing process by increasing gastric gel-forming macromolecular glycoprotein in the ulcerated region.

There is no doubt that the repair of the ulcerated region is accomplished by the regeneration of defective mucosa and by the repletion of the defective region through the formation of granulation tissue. In the present experiment, however, teprenone was ineffective in increasing the granulation tissue beneath the ulcer floor. We previously showed that in this ulcer model, the thickness of the ulcer base indicating the degree of granulation proliferation attained to a maximum on the 10th day and then reduced rapidly as time progressed up to the 40th day, while the mucosal regeneration index reached a maximum on the 40th day when the ulcer floor was completely covered with the regenerated mucosa. Therefore, it seems that it is not necessary for teprenone to stimulate the formation of granulation tissue beneath the ulcer floor more than the control in order to promote the ulcer healing at the resorbing stage of the granulation tissue after the 15 th day.

Cimetidine, a histamine $\mathrm{H}_{2}$ receptor antagonist used as a control drug, has a potent inhibitory action on gastric acid secretion and has been widely used for the treatment of gastric and duodenal ulcers in recent years. However, Kasajima et al. (11) endoscopically observed that protruded changes in the ulcer floor frequently occurred during the healing process in patients with gastric ulcer receiving cimetidine treatment. Furthermore, similar specific findings have also been shown after the treatment with other histamine $\mathrm{H}_{2}$ receptor antagonists such as ranitidine and famotidine $(12,13)$. In these reports, the biopsy specimen from the protruded lesion histopathologically showed granulation tissue which consisted of inflam- 
matory cell infiltration and renewed capillaries. In the present study, we also obtained results supporting the above mentioned findings. The mechanism for such a specific healing action of histamine $\mathrm{H}_{2}$ receptor antagonists still remains unclear. However, the specific healing action of these agents may be probably due to an inbalance between the regeneration of defective mucosa and the formation of granulation tissue beneath the ulcer floor. As observed with cimetidine in this experiment, excessive formation of granulation tissue beneath the ulcer floor may inhibit mucosa regeneration on the exposed ulcer floor.

Proglumide, a gastrin antagonist used as another control drug, has been demonstrated to possess not only a potent inhibitory action against gastric secretion (14) but also a defensive action of the gastric mucosa (15). However, in this experiment, proglumide at the dose used hardly showed any beneficial effect.

In the present study, teprenone showed the most potent promoting action among the three drugs used on the healing of acetic acid ulcer in rats. A long-term histological evaluation of the effects of teprenone alone and the combination with cimetidine is being studied by us and Nakazawa and his coworkers of the Second Department of Internal Medicine. Nagoya University School of Medicine.

Acknowledgement: We thank Dr. S. Nakazawa of the Second Department of Internal Medicine. Nagoya University School of Medicine, for his valuable advice on evaluating the histologica! findings.

\section{References}

1 Murakami, M., Oketani, K., Fujisaki, H., Wakabayashi, T. and Ohgo, T.: Antiulcer effect of geranylgeranylacetone, a new polysoprenoid on experimentally induced gastric and duodenal ulcers in rats. Arzneirnittelforsch. 31, 799-804 (1981)

2 Murakami, M., Oketani, K., Fujisaki, H., Wakabayashi, T., Ohgo, T. and Kawai, K.: Fundamental studies of the changes of gastric mucosubstances induced by cold-restraint stress in rats. J. Kyoto Pref. Univ. Med. 90, 443-451 (1981) (Abs. in English)

3 Murakami, M., Oketani, K., Fujisaki, H., Waka- bayashi, T., Ohgo. T. and Kawai, K.: Effects of geranylgeranylacetone on the changes of gastric mucosubstances in rats subjected to cold-restraint stress-Predictable evaluation as an antiulcer drug. J. Kyoto Pref. Univ. Mcd. 90, 453-459 (1981) (Abs. in English)

4 Oketani, K., Murakami, M., Fujisaki, H., Wakabayashi, T. and Hotta, K.: Effect of geranylgeranylacetone on aspirin-induced changes in gastric glycoproteins. Japan. J. Pharmacol. 33, 593-601 (1983)

5 Ito, M., Fujii, A. and Suzuki, Y.: Long-term histological observation of the healing process of acetic acid ulcer in rats and apparent relapse. in Experimental Ulcer-Research Trends in Japan. Edited by Tsuchiya, M., Oda, M. and Okazaki, I., p. 201-206, Yurinsha Ltd., Tokyo (1983)

6 Johnson, L.R. and Guthrie, P.D.: Proglumide inhibition of trophic action of pentagastrin. Am. J. Physiol. 246, G62-G66 (1984)

7 Takagi, K., Okabe, S. and Saziki, R.: A new method for the production of chronic gastric ulcer in rats and the effect of several drugs on its healing. Japan. J. Pharmacol. 19, 418-426 (1969)

8 Tabayashi, T.: Production of chronic ulcer in rats by clamping-cortisone method and histological findings. J. Gastroenterol. 62, 15331547 (1965) (in Japanese)

9 Murakami, M., Sasaki, Z., Misaki, F., Kawai, K. and Wakabayashi, T.: Study on the kinetics of mucus secreting cells in the gastrointestinal tract-Effects of various drugs and hormones on the cell kinetics of the generative zone in mouse gastric mucosa. Folia Pharmacol. Japon. 79, 591-597 (1982) (Abs, in English)

10 Fujii, A., Ito, M. and Suzuki, Y.: Changes in mucus high-molecular-weight glycoprotein amount and mucosal lysosomal enzyme activities during the healing process of acetic acid ulcer in rats. Japan. J. Pharmacol. 36, Supp. 362P (1984)

11 Kasajima, M., Morikawa, T., Kanayama, R., Takada, A. and Konishi, F.: Endoscopical and histological observations on the healing process of gastric ulcer with cimetidine treatment. Gastroenterol. Endosc. 23. 1746-1751 (1981) (Abs. in English)

12 lida, Y., Takeuchi, K., Harada, H., Tada, M., Yamaguchi, M., Saito, M., Miyazaki, S., Kawano, H., Nagatomi, Y., Harima, K., Fujita, K., Harima, T., Gotoh, T., Ohshita, Y., Hirata, M., Sakaki, N., Okazaki, Y. and Takemoto, T.: Endoscopic studies of healing process on gastric ulcer 
treated with ranitidine. Gastroenterol. Endosc 24, 1917-1925 (1982) (Abs. in English)

13 Harada, K., Yokota, K., Shirota, K., Hayashi, H., Kaji, I., Mizushima, K., Okamura, K. and Namiki, M.: Healing process of gastric ulcer showing protruding change treated with histamine $\mathrm{H}_{2}$ receptor antagonist. Gastroenterol. Endosc. 26 , 1481-1488 (1984) (Abs. in English)

14 Ishizaki, O., Saito, G. and Kagiwada, K.; The pharmacological study on proglumide (1) Effects of proglumide (KXM) on the gastric secretion and the peptic ulcers. Pharmacometrics 5, 185-192 (1971) (Abs. in English)

15 Moriga, $M$. and Aono, M.: Preventive and curative effects of proglumide on ulcerous lesions and mucosal hexosamine in aspirin-induced gastric ulcer of rats. Pharmacometrics 18, 173-179 (1979) (Abs. in English) 